\title{
Spoof surface plasmon waveguide forces
}

\section{Citation}

Woolf, David, Mikhail A. Kats, and Federico Capasso. 2014. "Spoof Surface Plasmon Waveguide Forces." Optics Letters 39 (3): 517. https://doi.org/10.1364/ol.39.000517.

\section{Permanent link}

http://nrs.harvard.edu/urn-3:HUL.InstRepos:41371345

\section{Terms of Use}

This article was downloaded from Harvard University's DASH repository, WARNING: This file should NOT have been available for downloading from Harvard University's DASH repository.

\section{Share Your Story}

The Harvard community has made this article openly available.

Please share how this access benefits you. Submit a story.

Accessibility 


\title{
Spoof surface plasmon waveguide forces
}

\author{
David Woolf, Mikhail A. Kats, and Federico Capasso* \\ School of Engineering and Applied Sciences, Harvard University, Cambridge, Massachussettes 02138, USA \\ ${ }^{*}$ Corresponding author: capasso@seas.harvard.edu
}

Received October 29, 2013; accepted November 16, 2013;

posted December 13, 2013 (Doc. ID 200260); published January 23, 2014

\begin{abstract}
Spoof surface plasmons (SP) are SP-like waves that propagate along metal surfaces with deeply sub-wavelength corrugations and whose dispersive properties are determined primarily by the corrugation dimensions. Two parallel corrugated surfaces separated by a sub-wavelength dielectric gap create a "spoof" analog of the plasmonic metal-insulator-metal waveguides, dubbed a "spoof-insulator-spoof" (SIS) waveguide. Here we study the optical forces generated by the propagating "bonding" and "anti-bonding" waveguide modes of the SIS geometry and the role that surface structuring plays in determining the modal properties. By changing the dimensions of the grooves, strong attractive and repulsive optical forces between the surfaces can be generated at nearly any frequency. (c) 2014 Optical Society of America
\end{abstract}

OCIS codes: (230.7400) Waveguides, slab; (240.6680) Surface plasmons; (160.3918) Metamaterials. http://dx.doi.org/10.1364/OL.39.000517

Surface plasmons (SPs) are transverse magnetic (TM)polarized waves that result from the coupling between photons and free electron oscillations in a metal at its interface with a dielectric and have been extensively studied over the past decade [1,2]. SPs in metal-insulatormetal (MIM) waveguides can lead to extreme subwavelength confinement [3], strong field enhancement [4], and even negative refraction [5] . Additionally, the field of active plasmonics has seen significant growth in recent years as researchers have attempted to integrate plasmonic elements into micro and nanoelectromechanical systems (MEMS and NEMS) for applications including optical circuitry [6], ultrafast optical switching [7], and optical trapping[]]. However, these studies have been limited almost entirely to the visible and near-infrared (IR) frequency ranges where materials like gold, silver, and transparent conducting oxides have strong plasmonic resonances $[9,10]$ but are also highly lossy. Subwavelength corrugations on the surfaces of metals allow for the extension of these concepts to lower frequencies where absorption losses in metals can be much smaller [11-15]. Corrugated surfaces support surface waves known as spoof surface plasmons (SSPs), whose behavior depends primarily on the geometry of the corrugations instead of the optical properties of the metal. The dispersion equation for SSPs on a corrugated perfect electric conductor (PEC) has been analytically calculated [12] as

$$
\frac{\kappa_{z}^{g}}{k_{0}}=\frac{a}{d} \tan \left(k_{0} \sqrt{\epsilon_{d}} h\right)
$$

where $k_{0}=2 \pi / \lambda_{0}, \lambda_{0}$ is the free space wavelength, $\kappa_{z}^{g}=i k_{z}^{g}=\sqrt{\beta^{2}-k_{0}^{2} \epsilon_{d}}$, where $k_{z}$ is the z-component of the wavevector in the gap and $\kappa_{z}^{g}$ is used to represent the evanescent quality of the wave, $\beta$ is the propagation constant in the x-direction, $\epsilon_{d}$ is the dielectric function of the bounding dielectric, set here to 1 to represent air, and $h, d$, and $a$ are defined in the Fig. 1(a) inset, with $d \ll \lambda_{0}$. The solution to Eq. (1) is shown in Fig. 1(a) for $h=2 \mu \mathrm{m}$, $d=0.5 \mu \mathrm{m}$, and $a / d=[0.002,0.2,0 . \overline{4,0.6}, 0.98]$ (solidblue to solid-red line). The single SSP dispersion curve can be understood as the "anticrossing" of a planewave dispersion curve and the horizontal line corresponding to a localized $\lambda / 4$ cavity resonance of a single groove at the so-called SSP frequency (wavelength), $\omega_{\mathrm{ssp}, 0}=\pi c / 2 h$ $\left(\lambda_{\text {spp }, 0}=4 h\right)$. Thus the SSP can be thought of as a very weakly bound mode when $\omega \ll \omega_{\mathrm{ssp}, 0}$ and a tightly bound (localized) mode when $\omega \approx \omega_{\mathrm{ssp}, 0}[\underline{13}, \underline{16}]$, with the transition between these two regimes becoming more gradual with increasing duty cycle $a / d$ [Fig. 1(b)].

Two corrugated metal surfaces separated by a dielectric gap create a metamaterial analog to the plasmonic MIM waveguide, which we refer to as a spoofinsulator-spoof (SIS) waveguide [Fig. 1(b)] $[\underline{13}, \underline{17}, \underline{18}]$.
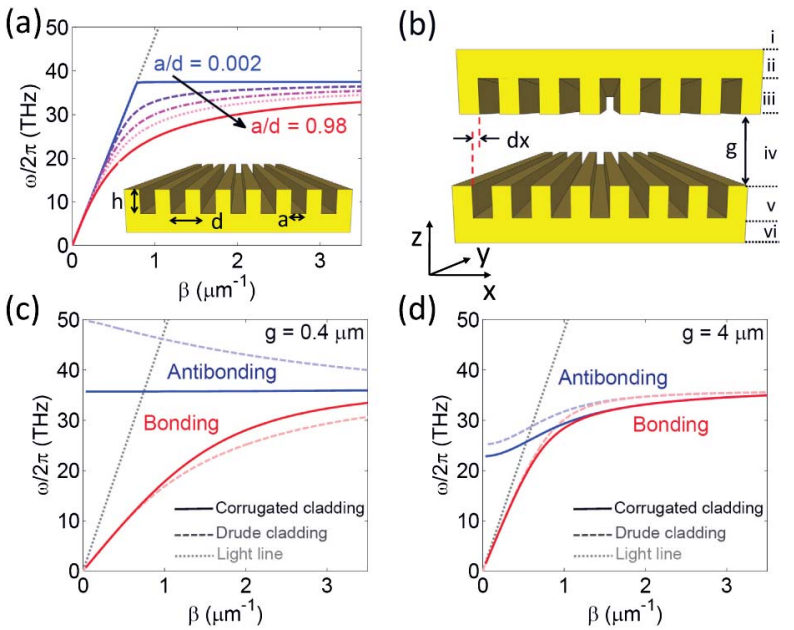

Fig. 1. (a) Dispersion relation of a single SSP mode, Eq. (1), for $h=2 \mu \mathrm{m}, d=0.5 \mu \mathrm{m}$, and $a / d=[0.002,0.2,0.4,0.6,0 . \overline{98}]$ (solid-blue to solid-red line), where $h, d$, and $a$ are defined in the inset. The black arrow represents increasing $a / d$. (b) Spoof-insulator-spoof (SIS) waveguide geometry consisting of a corrugated PEC cladding surrounding an air-gap core. Roman numerals I-VI correspond to the layers of the waveguide for dispersion calculations. (c), (d) Bonding (red) and antibonding (blue) modes in a SIS waveguide for (c) $g=0.4 \mu \mathrm{m}$ (d) and $g=4 \mu \mathrm{m}$. For comparison, the modes of an MIM waveguide consisting of artificial Drude metals with plasma frequency $\omega_{p}=\sqrt{2} \omega_{\text {ssp }, 0}$ are plotted as faded dashed lines in (c) and (d). The light line (gray-dotted line) is included for clarity. 
The dispersion equation for this geometry can be calculated using the transfer matrix method described in $[12,18]$, which requires us to calculate the reflectivity for light that is incident on the top of a five-layer stack defined by the Roman numerals II-VI in Fig. 1(b), which in turn requires us to define an effective permeability $\left(\epsilon_{s}\right)$ and permittivity $\left(\mu_{s}\right)$ of the corrugated layers of thickness $h$ (layers III, V) which are valid for TM modes. We do this using two simple heuristic arguments. First, we define the relative impedance $\eta_{r}=\sqrt{\mu_{s} / \epsilon_{s}}$ of the corrugated layer as the weighted average of the impedance of a PEC and air, equal to $a / d$. Second, we note that the grooves act as truncated parallel-plate waveguides in the z-direction, which support a single optical mode that follows the light line. In other words, light travels through the layer at $c$ (with $k_{z}^{s}=k_{0}$ ), implying that effective refractive index can be written as $n_{\text {eff }}=\sqrt{\epsilon_{s} \mu_{s}}=1$. From these two expressions, we can infer that $\epsilon_{s}=d / a$ and $\mu_{s}=a / d$, confirming the result first derived in [12].

Guided modes in the SIS geometry correspond to points with undefined values for the reflectivity $R$ of the stack known as removable singularities[18]. By setting the denominator of $R$ equal to zero, the following expression for the dispersion relation can be derived:

$$
\frac{\kappa_{z}^{g}}{k_{0}}=\frac{\epsilon_{d}}{\epsilon_{s}} \tan \left(k_{0} \sqrt{\epsilon_{d}} h\right) \tanh ^{ \pm 1}\left(\kappa_{z} g\right)
$$

where $g$ is the gap in the waveguide and the \pm corresponds to the bonding and antibonding modes, respectively. These modes are plotted in Figs. 1(c) and 1(d) as the red (bonding) and blue (antibonding) lines for the values of $h$ and $d$ given earlier and $a / d=0.5$. For comparison, we also plot the bonding and antibonding modes of a noncorrugated MIM waveguide (dashed lines), where we assumed a lossless Drude model for the metal cladding: $\epsilon_{\text {Drude }}=1-\omega_{p}^{2} / \omega^{2}, \omega_{p}=\sqrt{2} \omega_{\text {ssp }, 0}$.

For small gaps $[g=0.4 \mu \mathrm{m}$, Fig. 1(c)], SSPs in the bonding mode behave like the bonding-mode SPs in the MIM waveguide geometry, exhibiting strong dispersion and approaching the asymptote $\omega \rightarrow \omega_{\mathrm{ssp}, 0}$ as $\beta \rightarrow \infty$. Spoof plasmons in the antibonding mode, however, exhibit dispersive properties distinct from antibonding MIM-waveguide plasmons. As $g \rightarrow 0$, the SIS antibonding mode has nearly flat dispersion, corresponding to nearly zero group velocity, with $v_{g}=d \omega / d \beta \rightarrow 0^{+}$, whereas the MIM-waveguide antibonding mode has strong anomalous dispersion $(d \omega / d \beta<0)$ for these gap values. At large gaps [ $g=4 \mu \mathrm{m}$, Fig. $\underline{1(\mathrm{~d})]}$, the SIS waveguide modes and MIM waveguide modes are very similar, implying that the "plasmon-like" designation is valid in this limit. As $g \rightarrow \infty$ the bonding and antibonding modes approach degeneracy, where the only difference between the two modes is the relative phase of the waves on the two corrugated surfaces. The bonding mode corresponds to waves traveling along the two surfaces that are in-phase with one another, while the antibonding mode corresponds to waves that are out-of-phase with one another. We note that Eqs. (1) and (2) are valid only for SSPs on PECs when $\beta$ is less than $\pi / d$ by approximately a factor of two, since we can no longer treat the corrugated layer in the effective medium limit when the wavelength in the structure approaches the periodicity of the corrugations. Additionally, the preceding analysis ignored the losses that are present in systems involving real metals.

In order to account for all geometric and material effects, we use numerical techniques. We simulate a single unit cell of an SIS waveguide comprised of gold and air using the finite element method as implemented in COMSOL multiphysics, enforcing Floquet periodicity at the unit cell boundaries, which fixes the wavevector $\beta$ in each individual simulation. To account for material dispersion, we employ an iterative method to accurately determine the eigenfrequencies of the geometry. We start by solving Eq. (2) at a fixed $\beta$ to obtain $\omega_{0}^{b}$ and $\omega_{0}^{a}$, the initial guesses for the resonant frequencies of the bonding and antibonding modes, respectively and use these values to obtain $\epsilon_{\mathrm{Au}}\left(\omega_{0}^{b}\right)$ and $\epsilon_{\mathrm{Au}}\left(\omega_{0}^{a}\right)$ from the Drude model for gold $\left[\epsilon_{\mathrm{Au}}(\omega)=1-\omega_{p}^{2} /\left(\omega^{2}+i \gamma \omega\right)\right.$ with $\left.\omega_{p} / 2 \pi=2.18 e 15 \mathrm{~Hz}, \gamma / 2 \pi=4.34 e 12 \mathrm{~Hz}[\underline{19}]\right]$. We then solve in COMSOL for the bonding and antibonding modes separately, obtaining new eigenfrequency guesses, $\omega_{1}^{b}$ and $\omega_{1}^{a}$. We then repeat this process until the values of the eigenfrequencies converge [i.e., $\left(\omega_{n}^{b, a}-\omega_{n-1}^{b, a}\right) /$ $\omega_{n}^{b, a}<0.01$ ], for values of $\beta$ from $0.05 \pi / d$ to $0.55 \pi / d$. The resulting dispersion relations are plotted in Fig. 2(a) for $g=0.4 \mu \mathrm{m}$ (circles) and $g=4 \mu \mathrm{m}$ (squares).

Comparing Fig. 2(a) to Figs. 1(c) and 1(d), we find that the real-metal dispersion relations are qualitatively similar to the dispersion relations of SSPs on PECs, though the differences are noteworthy. First, light inside the grooves does not propagate at $c$ (i.e., $k_{z}^{s} \neq k_{0}$, as we assumed earlier). Instead, the grooves act as short MIM waveguides of gap $a$ and have a corresponding effective mode index, $n_{\text {eff }}>1$ [20,21], which changes the cavity resonance condition. Second, the periodicity of the structure introduces a band edge at $\beta_{\text {edge }}=\pi / d$ causing the effective material assumption to break down [12] and resulting in $\omega_{\text {ssp }}\left(\beta_{\text {edge }}\right)<\omega_{\text {ssp }, 0}$. We note that our calculations also show a negligible dependence on the lateral offset $d x$ between the corrugations on the two surfaces, confirming that the structures are in the effective medium limit.

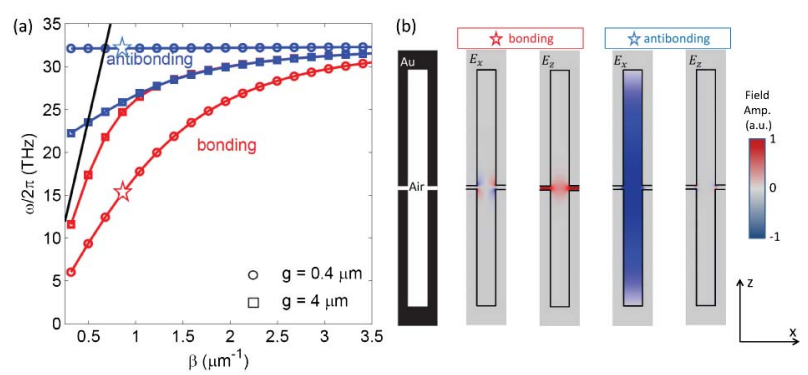

Fig. 2. (a) Dispersion curves for the bonding (red lines) and antibonding (blue lines) modes of the SIS geometry simulated with corrugated gold surfaces corresponding to the geometry in Fig 1(a). Modes were solved at $g=0.4 \mu \mathrm{m}$ (circles) and $g=4 \mu \mathrm{m}$ (squares), corresponding to the parameters in Figs. 1(c) and 1(d). (b) Electric field profiles of a unit cell of the structure for the bonding and antibonding modes at $g=0.4 \mu \mathrm{m}$ and $\beta=1 \mu \mathrm{m}^{-1}$. 
Simulations of the field profiles [Fig. 2(b)] at $g=$ $0.4 \mu \mathrm{m}$ and $\beta=1 \mu \mathrm{m}^{-1}$ illustrate the differences between the two modes. The bonding mode is well confined within the small gap between the two corrugated surfaces and is dominated by the z-component of the electric field, similar to a parallel plate capacitor or the TEM mode of a parallel-plate waveguide. As in a capacitor, the surface charge distribution (and $E_{\|}=E_{x}$ ) with respect to the center of the gap is antisymmetric while $E_{\perp}=E_{z}$ is symmetric and corresponds to an attractive force between the surfaces. Light in the antibonding mode, on the other hand, has antisymmetric $E_{\perp}$ and a symmetric charge distribution with respect to the gap, resulting in a repulsive force.

We can calculate the force between the two surfaces by integrating the Maxwell stress tensor across a plane separating the two surfaces [22]. We plot the magnitude of the force $\left|F_{z}\right|$ as a function of $g$ in Fig. 3(a) for the bonding (red lines) and antibonding modes (blue lines) at $\omega / 2 \pi=30 \mathrm{THz}(\lambda=10 \mu \mathrm{m}$, solid lines $), 27 \mathrm{THz}$ (11 $\mu \mathrm{m}$, dashed lines), and $25 \mathrm{THz}(12 \mu \mathrm{m}$, dashed-dotted lines), normalizing the force per unit area to the power flowing through the waveguide. At large gaps $(g>\lambda / 4)$, the bonding and anti-bonding forces have approximately the same magnitude but opposite sign, corresponding to gaps where the dispersion curves approach degeneracy [Fig. 3(b)]. As the gap decreases, the splitting between the modes increases and the bonding and antibonding force magnitudes diverge. The repulsive force generated by the antibonding mode grows asymptotically as the separation approaches the value corresponding to cutoff for the mode $g_{c} \equiv g(\beta=0) . g_{c}$ corresponds to the lowest order mode of a vertical cavity defined by the region between the bottom of a groove on the bottom surface and the top of a groove on the top surface. For small gaps, the cutoff wavelength is approximately given by $\lambda / 2 \approx 2 h+g$. The force generated by the bonding mode increases exponentially as the gap width decreases, corresponding to an increase in field intensity in the gap, and the build-up of surface charge, mimicking the properties of the MIM waveguide bonding mode [19]. These forces are slightly larger than those reported in an earlier work on MIM and IMIMI modes[19], and on par with what has
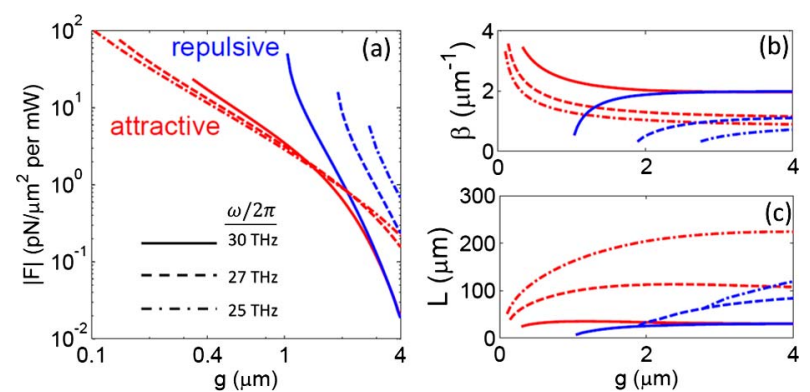

Fig. 3. (a) Attractive (red lines) and repulsive (blue lines) forces generated by the bonding and antibonding modes, respectively, at three wavelengths: $\omega / 2 \pi=30 \mathrm{THz}$ (solid lines), $27 \mathrm{THz}$ (dashed lines), and $25 \mathrm{THz}$ (dashed-dotted lines), as a function of gap width. (b) Wavevectors of these modes showing degeneracy at large gaps and splitting at small gaps. The antibonding mode has a clear cutoff gap. (c) Propagation length of the bonding (red) and antibonding (blue) modes plotted in (a). been theoretically reported in parallel dielectric waveguides[23] at visible and near-IR frequencies.

Applications of these structures, like conventional SP waveguides, will ultimately be limited by absorption. MIM waveguides that achieve subwavelength confinement have propagation lengths on the order of only one free-space wavelength at visible and near-IR frequencies, introducing significant heating and limiting the possibilities for optical interconnects or active-plasmonic devices at these frequencies [3]. Other options for mid-IR surface waveguiding, such as surface phonon polaritons, suffer from significant absorption due to their proximity to a material resonance [24]. Efficient terahertz waveguiding can be achieved with heavily doped semiconductors [25]; these structures obtain slightly better propagation lengths than spoof structures but do not support antibonding modes at small gaps.

Given a lossy system, both the frequency and the wavevector can be complex. It is typical to assume that one of these is well-defined experimentally and therefore purely real. In these calculations we assume a well-defined wavevector $\beta$ (corresponding to excitation by a grating etched into one side of the waveguide, for example), and calculate the corresponding complex frequency $\tilde{\omega}=\omega^{\prime}+i \omega^{\prime \prime}$, which gives us both the real dispersion relation and the waveguide losses [26]. We quantify the absorption in our modes by calculating the propagation length $L=-2 v_{g} / \omega^{\prime \prime}$ and plotting it in Fig. 3(c) for the modes in Fig. 3(b). As expected, the propagation lengths of modes approach degeneracy at large gaps and are longer for wavelengths that are farther from the SSP resonance. However, simulations reveal that the bonding mode experiences a peak in its propagation length at an intermediate gap width that approximately corresponds to $g_{c}$ of the antibonding mode [see Fig. 3(b)]. This result is counterintuitive, as one would expect that a more confined mode would propagate slower and experience more loss due to field overlap with the metal surfaces. The increase in $L$ is the consequence of an increase in $v_{g}$, which also occurs in SIS waveguides formed with PECs and standard MIM waveguides, according to our analytical calculations, though to our knowledge is thus far unreported. For $\lambda_{0}<11 \mu \mathrm{m}$, propagation lengths of longer than $10 \lambda_{0}$ are achievable with $g \ll \lambda_{0}$, making this a viable geometry for waveguiding applications.

Experimental realization of these structures will be necessary to demonstrate a new array of optomechanical devices at mid-IR and terahertz frequencies. While single SSP waveguides have been demonstrated $[14,16,17]$, none have truly been in the metamaterial limit $(d<\lambda / 10)$, as the aspect ratio of the grooves required to reach this limit is difficult to achieve experimentally. However, structures equivalent to spoof surfaces have been demonstrated by placing a periodic array of metallic elements, such as patch antennas [27], split-ring resonators [28], or potentially V [29], Y [30], or H-shaped [31] antennas separated from a metallic backplane by a thin dielectric layer. Instead of controlling the depth of the grooves to determine the SSP resonance, the shape of the metallic elements changes the phase response of the surface, and thus also controls the resonance condition. 
In conclusion, we have described the dispersion of SSP modes in SIS waveguides, demonstrating that MIM waveguiding concepts can be extended into the mid-IR and terahertz frequency ranges where losses are comparatively smaller. We investigated the optical forces generated by SSPs and showed that forces greater than radiation pressure are attainable for highly confined mid-IR optical fields. The attractive and repulsive forces generated within SIS waveguides have a wide range of potential applications, as they extend optomechanical functionality to any frequency range while maintaining tight confinement of the optical field.

We thank N. Yu, S. Byrnes, and R. Blanchard for their helpful discussions. We acknowledge support from AFOSR under contract no. FA9550-09-1-0505. M. A. Kats is supported by the NSF Graduate Research Fellowship.

\section{References}

1. S. Lal, S. Link, and N. J. Halas, Nat. Photonics 1, 641 (2007).

2. S. A. Maier and H. A. Atwater, J. Appl. Phys. 98, 011101 (2005).

3. H. T. Miyazaki and Y. Kurokawa, Phys. Rev. Lett. 96, 097401 (2006).

4. H. T. Miyazaki and Y. Kurokawa, Appl. Phys. Lett. 89, 211126 (2006).

5. H. J. Lezec, J. A. Dionne, and H. A. Atwater, Science 316, 430 (2007).

6. W. Cai, W. Shin, S. Fan, and M. L. Brongersma, Adv. Mater. 22, 5120 (2010).

7. K. F. MacDonald, Z. L. Samson, M. I. Stockman, and N. I. Zheludev, Nat. Photonics 3, 55 (2008)

8. X. D. Yang, Y. M. Liu, R. F. Oulton, X. B. Yin, and X. A. Zhang, Nano Lett. 11, 321 (2011).

9. W. A. Murray and W. L. Barnes, Adv. Mater. 19, 3771 (2007).

10. C. Rhodes, S. Franzen, J. P. Maria, M. Losego, D. N. Leonard, B. Laughlin, G. Duscher, and S. Weibel, J. Appl. Phys. 100, 054905 (2006).

11. J. B. Pendry, L. Martin-Moreno, and F. J. Garcia-Vidal, Science 305, 847 (2004).
12. F. J. Garcia-Vidal, L. Martin-Moreno, and J. B. Pendry, J. Opt. A 7, S97 (2005).

13. B. W. Wang, Y. Jin, and S. L. He, Appl. Opt. 47, 3694 (2008).

14. N. F. Yu, Q. J. Wang, M. A. Kats, J. A. Fan, S. P. Khanna, L. H. Li, A. G. Davies, E. H. Linfield, and F. Capasso, Nat. Mater. 9, 730 (2010).

15. A. Pors, E. Moreno, L. Martin-Moreno, J. B. Pendry, and F. J. Garcia-Vidal, Phys. Rev. Lett. 108, 223905 (2012).

16. J. Zhang, L. K. Cai, W. L. Bai, Y. Xu, and G. F. Song, J. Appl. Phys. 106, 103715 (2009).

17. K. Song and P. Mazumder, IEEE T. Electron. Dev. 56, 2792 (2009).

18. M. Kats, D. Woolf, R. Blanchard, N. Yu, and F. Capasso, Opt. Express 19, 14860 (2011).

19. D. Woolf, M. Loncar, and F. Capasso, Opt. Express 17, 19996 (2009).

20. A. Rusina, M. Durach, and M. I. Stockman, Appl. Phys. A 100, 375 (2010).

21. X.-R. Huang, R.-W. Peng, and R.-H. Fan, Phys. Rev. Lett. 105, 243901 (2010)

22. J. D. Jackson, Classical Electrodynamics (Wiley, 1999).

23. M. L. Povinelli, M. Loncar, M. Ibanescu, E. J. Smythe, S. G. Johnson, F. Capasso, and J. D. Joannopoulos, Opt. Lett. 30, 3042 (2005).

24. A. Huber, N. Ocelic, D. Kazantsev, and R. Hillenbrand, Appl. Phys. Lett. 87, 081103 (2005).

25. A. Degiron and D. R. Smith, Opt. Express 14, 1611 (2006).

26. V. M. Agranovich, Surface Polaritons (Elsevier, 1982).

27. M. J. Lockyear, A. P. Hibbins, and J. R. Sambles, Phys. Rev. Lett. 102, 073901 (2009).

28. M. Navarro-Ca, M. Beruete, S. Agrafiotis, F. Falcone, M. Sorolla, and S. A. Maier, Opt. Express 17, 18184 (2009).

29. N. Yu, P. Genevet, M. A. Kats, F. Aieta, J.-P. Tetienne, F. Capasso, and Z. Gaburro, Science 334, 333 (2011).

30. M. A. Kats, P. Genevet, G. Aoust, N. Yu, R. Blanchard, F. Aieta, G. Gaburro, and F. Capasso, Proc. Natl. Acad. Sci. USA 109, 12364 (2012).

31. S. Sun, Q. He, S. Xiao, Q. Xu, X. Li, and L. Zhou, Nat. Mater. 11, 426 (2012). 